Cahiers québécois de démographie

\title{
Les démographes et la population du Canada sous le régime
} français (1934-1966)

Demographers and the Population of Canada under the French Regime (1934-1966)

\section{Los demógrafos y la población de Canadá durante el régimen francés (1934-1966)}

\section{André LaRose}

Volume 13, numéro 1, avril 1984

Population et histoire

URI : https://id.erudit.org/iderudit/600520ar

DOI : https://doi.org/10.7202/600520ar

Aller au sommaire du numéro

Éditeur(s)

Association des démographes du Québec

ISSN

0380-1721 (imprimé)

1705-1495 (numérique)

Découvrir la revue

Citer cet article

LaRose, A. (1984). Les démographes et la population du Canada sous le régime français (1934-1966). Cahiers québécois de démographie, 13(1), 41-57.

https://doi.org/10.7202/600520ar
Résumé de l'article

Avant que les démographes Hubert Charbonneau et Jacques Légaré ne lancent leur vaste enquête sur la population de la Nouvelle-France au milieu des années soixante, d'autres démographes s'étaient penchés sur l'histoire de cette même population : Georges Langlois en 1934, Georges Sabagh en 1942, Jacques Henripin en 1954 et Jean-Noël Biraben en 1966. On trouvera ici un compte rendu de leurs travaux. Après avoir présenté l'une après l'autre leurs publications respectives, l'auteur évalue leur contribution et met en relief les forces et faiblesses de chacun, en abordant tour à tour les sources et la critique des sources, l'état de la population et sa structure, le mouvement de la population et ses composantes et enfin, les idées et les politiques démographiques. 
Cahiers québecois de demographie

Vol. 13, no 1 , avril 1984

\title{
Les démographes et la population du Canada sous le régime français (1934-1966)
}

\author{
André LaROSE*
}

\begin{abstract}
L'etude de la population est un champ d'interet que se partagent plusieurs disciplines: la genéalogie, l'histoire, la gégraphie et la démographie, entre autres. Les historiens ne sont done pas les seuls a faire evoluer l'historiographie de la population et, dans le cas de la Nouvelle-France, en particulier, l'apport des démographes mérite d'etre souligné. Precisons au départ qu'il faut distinguer deux periodes dans la contribution des démographes a l'etude de la population canadienne aux XVIIe et XVIIIe siecles: l'une qui va de 1934 a 1966; 1'autre qui va de 1966 a nos jours. La premiere est jalonnee de publications de Georges Langlois, Georges Sabagh, Jacques Henripin et JeanNoël Biraben; la seconde est dominee par une equipe de chercheurs du Département de démographie de l'Université de Montrél rénis autour d'Hubert Charbonneau et de Jacques Légaré, sous le nom de Programe de recherche en dénographie historique.
\end{abstract}

Les annés 1934 et 1966 s'imposent en effet comme points de repere: 1934 est l'année où a été publiée l'Histoire de la population canadienne-francaise de Georges Langlois, un ouvrage dans lequel Jacques Henripin a vu "l'acte de naissance de la recherche demographique sur le Canada français" (Henripin, 1962: 133); quant à 1966, c'est l'année où Charbonneau a fait paraître une note de recherche intitulee "Tricentenaire du premier recensement canadien", breve etude qui devait être le prélude a une vaste enquete dont les bases ont eté posés dans une communication présentée au congres de l'ACFAS en 1966 (Charbonneau et Légaré, 1966; Charbonneau et al., 1967). L'envergure des travaux qui ont ete entrepris depuis par le programme de recherche en démographie historique étant sans commune mesure avec celles des travaux réalisés précédemment, et les publications de l'equipe etant nombreuses et diversifiés, il nous a paru préférable de laisser de coté cette abondante production et de ne rendre compte ici que des travaux anterieurs. C'est donc un compte rendu des travaux des demographes qui ont ecrit entre 1934 et 1966 sur la population de la Nouvelle-France que nous présentons ici. Nous nous proposons d'abord de suivre dans le temps l'evolution de la connaissance en résumant la démarche de chacun des auteurs; ensuite, nous analyserons le contenu de leurs oeuvres en empruntant a la démographie son decoupage par theme, ce qui nous permettra de situer les contributions les unes par rapport aux autres ainsi que de les evaluer.

* Departement d'histoire, Université d'Ottawa. 


\section{PRÉSENTATION DES OEUVRES}

L'Histoire de la population canadienne-française est une these que Georges Langlois a faite a l'Universite de Paris sous la direction de René Gonnard, spécialiste des doctrines économiques et des doctrines de population. "Modeste monument élevé a la gloire du peuple canadien-français, resté français malgré l'Angleterre et loin de la France" (1935: dédicace), l'ouvrage de Langlois est en quelque sorte le fruit d'un travail d'inventaire, fait avec méthode, sous la poussée du sentiment national. L'auteur, qui n'aurait pu mener a bien pareille entreprise sans un minimum de formation en demographie, est frappé conme bon nombre de ses prédécesseurs et contemporains par le phénomene de la survivance française au Canada. A la différence de ceux-ci, qui "se bornent a constater le fait de cette survivance" quand ils ne font pas qu'en parler avec verbosité (1935: 2), Langlais tente dans son ouvrage d'expliquer cette situation dont le facteur essentiel est, selon lui, "la population, ce point central d'ou rayonnent et vers lequel convergent toutes les autres activités saciales" (1935: 2). L'auteur ne manque pas, au passage, de décocher quelques fléches aux historiens qui se sont limités au politique ou qui ont "fait des incursions dans les domaines juridique, économique, litteraire, religieux" sans se soucier de l'interaction entre la population et les institutions (1935: 2). Mais, c'est sans prétention que Langlois tente de redonner au facteur démographique la place qui lui revient dans "l'equation historique". Devant l'ampleur de la tache, il ne vise qu'a faire un premier pas dans le sens de l'exploration sommaire" qui doit préceder les analyses et l'etude definitive (1935: 4).

L'ouvrage se divise en deux parties - fort inégales - la preniere, consacree a l'histoire de la population de 1608 a 1930 (13 chapitres) et la seconde, a un essai sur les doctrines et les politiques de population du XVIle au XIXe siecles (4 chapitres). Le régime français y occupe d'ailleurs une place preponderante, l'auteur trouvant deux fois plus a dire sur cette période (115 p.) que sur les cent soixante-dix ans qui suivirent la Conquete (55 p.). La mene prééminence se retrouve du reste dans la seconde partie. Des sept chapitres consacrés a la Nouvelle-France dans la premiere partie de l'Histoire de la population canadienne-francaise, les quatre premiers donnent, selon un plan chronologique, un aperçu de l'évolution de la population. Langlois s'attache d'abord a l'immigration française dans la vallée du Saint-Laurent, avant de s'interesser a l'accroissement de cette petite population, a sa composition par age, par sexe et par etat matrimonial de meme qua sa repartition géographique. Viennent ensuite trois chapitres portant sur des themes précis: la nuptialité, la natalité et la mortalité. Dans l'ensemble, le XVIle siecle est abondamment traité et le XVIIIe siecle, plutat néglige. Dans la seconde partie de l'ouvrage, Langlois essaie d'expliquer les eléments de la politique française de population a l'égard de la Nouvelle-France (comment peupler la colonie sans depeupler la Francel, en les situant dans le contexte du mercantilisme et du populationnisme. L'auteur s'engage ensuite dans une comparaison entre les politiques françase et anglaise de colonisation, en insistant surtout sur les differences entre les deux entreprises. Il acheve son essai par un eloge du systeme seigneurial, cadre du developpement démographique. 
Georges Langlois a aussi ecrit un article qui concerne, de loin, la population du Canada sous le régime français. Il s'agit d'un "Essai sur le peuplement de l'Amérique" que l'auteur présente en sous-titre comme une "Introduction a une etude de la population du Canada" (1938 et 1939). L'auteur y situe le peuplement de la vallee du Saint-Laurent dans le cadre du peuplement du Nouveau Monde par l'Ancien et compare ce phenomene au peuplement des colonies americaines, en insistant sur les causes des mouvements migratoires.

En 1942, un sociologue americain a a son tour manifesté de l'interet pour la population canadienne du XVIIe siecle. Georges Sabagh s'est alors demandé si les femmes jouissaient a cette epoque d une fécondite exceptionnelle. Il repond par l'affirmative, en s'appuyant pour ses calculs sur les recensements de 1666,1667 et 1681 , publies par Benjamin Sulte a la fin du XIXe siecle. Il obtient des taux de fecondité éleves parce que la plupart des femmes en age de procreer a cette epoque etaient mariées. Il explique ces résultats par le fait que l'Eglise et l'Etat favorisaient les mariages hatifs et les familles nombreuses et que, dans un pays neuf, on avait besoin de bras pour cultiver la terre et se defendre contre l'Indien ou l'Anglais.

Vint ensuite la population canadienne au debut du xVIIle siecle de Jacques Henripin, these dans laquelle l'auteur a eté le premier a tenter de mesurer en profondeur la nuptialité, la fécondité et la mortalite infantile dans cette population. L étude, qui a eté menee dans le cadre de l'Institut National d'Etudes Jénographiques a Paris et publiée en 1954 par cet organisme, rend compte des préoccupations des demographes de l'époque - et plus spécialement de celles de Louis Henry - au sujet de la fecondité naturelle. Au debut des années cinquante, en effet, le lent affaissement de la fécondité en Europe, de 1801 a 1943, puis son brusque redressement par la suite ont de quoi intriguer les démographes. Il leur faut donc s'interroger sur les facteurs biologiques de la fécondité et, pour ce faire, observer des populations ou la limitation des naissances est inconnue ou peu pratiquee. Comme les populations du Tiers-Monde contemporain ne s'y pretent guere parce que l'enregistrement démographique y fait defaut, ils sont forcés de se rabattre sur des populations europeennes ou de souche européenne du passé pour lesquelles un tel enregistrement existe, grace aux registres paroissiaux anciens ou aux généalogies qui en ont été tirées (Henry, 1973: 341-342). C'est ce que fait Henripin dans sa these, qui s'appuie sur une exploitation ingenieuse du Dictionnaire genéalogique des fanilles canadiennes de Cyprien Tanguay; c'est aussi ce que fait Louis Henry dans ses Anciennes falliles genevoises (1956).

L'étude d'Henripin donne des eléments de mesure sur l'accroissement d'une population soumise au jeu des forces de la nature. Son grand merite est de jeter un peu de lumiere sur la question de la fécondité naturelle. Or, Henripin est le premier a étudier ce phenomene pour une population aussi ancienne et ses resultats confirment meme la these de Malthus qui dit qu'en régime naturel, une population double ses effectifs tous les vingt ou vingt-cing ans. En un sens, on peut dire que l'ouvrage dépasse son objet puisque, comme l'ecrit Alfred Sauvy dans sa presentation (1954: xii), 


\begin{abstract}
"Non seulement nous obtenons des résultats inedits, probants et utiles sur la population étudié, mais nous trouvons aussi un ensemble d'enseignements de caractere plus géneral sur l'aptitude de l'espece humaine a se multiplier ou se perpétuer, enseignements $s i$ puissants qu'il serait mal venu de les condiderer comme un simple sous-produit de ces travaux."
\end{abstract}

On comprend alors que l'ouvrage se soit acquis une réputation internationale et qu'il soit vite devenu un classique de la demographie. Signalons ici qu'Henripin a résume le fruit de ses recherches pour les lecteurs de population (1954b): dans son article, il traite de la fécondité légitime des couples suivant l'age de la femme, l'age au mariage et la durée de vie conjugale. L'article s'achève sur quelques élements de réponse au probleme de la stérilité liée à l'allaitement.

Quelques annés plus tard, dans le premier chapitre de son ouvrage sur les Tendances et facteurs de la fecondite au Canada, Henripin présentait une "Brève histoire démographique de la Nouvelle-France (1608-1760) et de la population catholique du Québec, de 1760 a 1880" (1968: 1-14). L'auteur y retrace en gros l'évolution de la population de la vallée du Saint-Laurent. Un bref examen des taux de nuptialité, de natalité et de mortalité aide alors a comprendre ce mouvement. Henripin reprend ensuite les grandes lignes de sa these sur la fécondité aux XVIIe et XVIIIe siecles. Puis il attire l'attention sur la soumission de cette population a la nature et aux évenements en donnant les exemples des epidemies - qui firent de nombreuses victimes - du mouvement saisonnier des naissances, et des guerres - qui jouent aussi bien sur les naissances que sur les décès. Il conclut en dégageant trois grandes caracteristiques de la croissance de la population canadienne-française: l'absence de grandes catastrophes (famines, epidémies et guerres), une nuptialité relativement forte, et, enfin, le maintien d'une forte fécondité des couples (1968: 14).

Il existe un troisieme essai de synthese d'histoire de la population canadienne-française. Il s'agit d'une communication du docteur Jean-Noël Biraben (1966) a la Société de démographie historique intitulée "Le peuplement du Canada français". Le texte se divise en trois parties: la premiere porte sur le peuplement primitif, c'est-a-dire l'occupation progressive de l'Amérique du Nord par les Amerindiens; la seconde, sur le peuplement francais jusqu'en 1760; enfin, la troisieme traite du peuplement depuis l occupation anglaise et l'auteur y parle non seulement des Québécois mais aussi des francophones de l'Ouest, de l'Ontario et des Maritimes. La premiere partie compte sept pages et demie et les deux autres, douze pages et demie chacune, tableaux, cartes et graphiques inclus. Toutes proportions gardés, c'est donc le régime français qui a la place d'honneur.

Aprés avoir parlé des essais de peuplement français au XVIe siècle, Biraben suit l'évolution du peuplement canadien au XVIIe siècle, avec une attention spéciale a la période 1663-1672, étant donné son intéret marqué pour la question de l'origine des immigrants et des filles a marier. L'auteur 5 arrete a 1720 dans son exposé chronologique; il résume alors la situation de 
l'immigration en Nouvelle-France et donne les principales caracteristiques de la population canadienne de lepoque avant de traiter des Pays-den-haut et de l'Acadie.

La synthese de Biraben est originale; l auteur envisage son sujet de façon plus large que Langlois ou Henripin dans sa "Ereve histoire demographique". Ses horizons géographiques sont moins limités: lauteur présente de façon nouvelle la population des Pays-d'en-haut; il situe le peuplement du Canada français dans le contexte des relations internationales de l'epoque: la prise de Quebec, I'Acadie qui change de main, l envoi des filles a marier non seulement au Canada mais aussi dans dautres colonies françaises; enfin, et surtout, il étudie les rapports du peuplement francais avec ses voisins amerindiens et anglo-saxons: les Français apportent la rougeole, le typhus et la variole aux Indiens, mais sont melés a la querre entre Hurons et Iroquois; les Hollandais, les Ecossais et les Anglais s'installent en Amerique au début du XVIle siecle, les Anglais harcelent les Acadiens au point de les déporter (1966: 113, 116, 121-122). La premiere et la troisieme partie de la communication sont cependant plus eloquentes que la seconde sur ces rapports.

\section{ANALYSE DE CONTENU PAR THÈMES}

\section{Les sources et la critique des sources}

L une des caracteristiques des travaux que nous analysons ici est qu'aucun d'eux ne s'appuie sur des sources de premiere main. Recensements et registres paroissiaux originaux ont ete ignores, au profit des sources imprimés, telles les séries statistiques sur letat et sur le mouvement de la population (Canada, 1876: 2-62; 1878: 160-267), ainsi que des sources secondaires, comme le Dictionnaire Tanguay, les ouvrages de Salone, Sulte et Ferland (pour ne citer que ceux-la), ou encore la documentation sur les immigrants accumulee par Archange Godbout'. A l'epoque où les auteurs etudiés ont écrit, les

1 Le Dictionnaire genealogique des fanilles canadiennes de Cyprien Tanguay est un ouvrage en sept volumes publie entre 1871 et 1890 . L'auteur $y$ a reconstitué les familles qui ont vécu dans la vallée du Saint-Laurent depuis les débuts de la colonie jusqu'au milieu du XVIIIe siecle environ. Dans une oeuvre intitulée "Nos Ancetres au XVIIe siecle", publiée par tranche dans le Rapport de I'Archiviste de la Province de Quebec entre 1951 et 1965, Archange Godbout a en quelque sorte repris une partie de l oeuvre de Tanguay, de façon plus detaillé; la mort l'a cependant empeché d'achever son oeuvre, dont la publication sarrete au patronyme Brassard. Les manuscrits de Godbout sont toutefois conservés aux Archives nationales du Québec, a Quebec. 
archives sur l'histoire de la population étaient dispersees et difficiles dacces et, aux yeux des démographes, il ne semblait pas necessaire d'y recourir. Langlois, par exemple, qui na pas voulu se "flatter dutiliser des documents de premiere main" déjà compulsés par les historiens, écrit:

"Il nous a paru suffisant dappuyer nos affirmations sur les auteurs qui ont, eux, utilisé les documents originaux et dont les travaux ont une autorité reconnue, de confronter leurs données lorsqu'elles netaient pas concordantes pour choisir la plus acceptable." (1935: 271)

['est donc chez les historiens qu'il a lus qu'il puise entre autres des témoignages d'epoque montrant que les familles avaient beaucoup denfants au XVIIe siecle. Et comme ces auteurs traitent davantage de la Nouvelle-France que du regime anglais ou des soixante premieres annés de la Confédération, on comprend que Langlois accorde finalement plus d'importance a l histoire de la population davant 1760 et quil soit obligé de miser sur les statistiques officielles et sur ses connaissances en démographie plus que sur les auteurs lorsqu il traite des annés 1760-1930. Sabagh, lui, fonde son etude sur les recensements du XVIIe siecle publies par Benjamin Sulte. Henripin, pour sa part, en tirant parti du Dictionnaire genealogique des fanilles canadiennes, fait d un ouvrage destiné la connaissance des individus un instrument de la connaissance de la collectivité. Quant a Biraben, c'est surtout du côté du fonds Godbout quil se tourne, question de renouveler le chapitre de l'immigration en Nouvelle-France. Cela dit, on doit aussi se demander quelle place accordent nos auteurs a la critique des sources. L'examen de leurs publications montre en fait que, Henripin mis a part, cet aspect de la recherche n'a pas suffisamment retenu l'attention des demographes avant 1966.

On constate notamment que le manque desprit critique a fait commettre quelques erreurs a Langlois. En voici deux exemples: dans le premier cas, la population de la Nouvelle-Beauce est passée de 245 en 1739 a 132 en 1744 , puis a 166 en 1751 (1935: 86). N'est-ce pas plutót le recensement qui peche par défaut? Il suffirait de calculer les taux de natalité et de mortalité dans les paroisses concernees pour s'en rendre compte. Dans le second cas, la comparaison de la natalité et de la mortalité a Boucherville, a Cap-Santé et a 1'Ile-aux-Coudres (1935: 125-126) - qui vient tout droit de Salone (1905 et 1970: 354-355) - nous laisse perplexe. De 1741 \& 1754, il n'y a que deux ans où l'on aurait enregistré plus de dix naissances a Boucherville, et six ans (dont trois annés consécutives) ou personne ne serait mort: c'est invraisemblable. Encore faut-il que ces trois paroisses soient de dimension comparable!

Sabagh non plus ne pousse pas tres loin l'esprit critique. "The relative reliability of the early censuses seems to be well established" ecrit-il (1942: 682). Les raisons invoquees sont des raisons de principe - il sagit de recensements nominatifs de jure, ils ont lieu a une date précise ("a fixed 
date"12 et les caractéristiques des individus sont enumérées. Il ajoute qu'on peut s'y fier à cause du zele de Talon et de Duchesneau a satisfaire aux demandes rovales. Quant a l état civil, sa valeur a été vérifiee par kuczynskis - cet argument dautorite lui suffit.

Biraben, pour sa part, nest quere éloquent sur les sources qu'il utilise, ce qui rend plus difficile la critique des chiffres qu'il rapporte - sur les filles a marier, par exemple. A vrai dire, le tableau des "Jeunes filles envoyés au Canada pour marier les soldats" (1966: 117) est assez confus: il met côte a côte des donnees concernant toute la France et dautres qui ne touchent qu'une partie de celle-ci; peut-etre l'auteur a-t-il voulu souligner de cette façon le desaccord entre les auteurs. Nous avons également relevé un certain nombre derreurs de calcul dans les tableaux des pages 117,118 et 119.

Au fond, la premiere critique sérieuse des sources que nous ayons est celle d'Henripin sur le Dictionnaire Tanguay. Lauteur consacre en effet un chapitre de sa these a l'exposé des sources et a leur critique, avant d'expliquer sa méthode (1954a: 25-38). Henripin decrit le travail de Tanguay et les difficultés qu'il a rencontrees avant de présenter le Dictionnaire et les erreurs inherentes a cette oeuvre: attribution denfants a leurs grands-parents plutot qua leurs parents, double inscription d'une meme famille sous deux noms avec un nombre different denfants aux deux endroits, omission d'enfants dans une famille, etc. L'auteur va mene jusqua vanter les mérites de son instrument, car

\begin{abstract}
"L'application de la table de fécondité trouvé montre qu on serait mal venu de reprocher a l'utilisation du Dictionnaire de Mgr Tanguay une sous-estimation de la fécondité, ce qui, a priori, etait a craindre, en raison des omissions existant fatalement dans un tel document." (1954a: 72)
\end{abstract}

Reste voir quel parti les auteurs ont tiré de leurs sources pour nous faire connaitre le nombre des Canadiens aux XVIIe et XVIIIe siecles.

2 Des recherches ont demontré par la suite que le recensement de 1666 avait été effectué entre le début de février et la find'août, que celui de 1667 s'était echelonné de la fin d'avril a la fin d'octobre et qu'enfin celui de 1681 sétait déroulé de la fin de juillet a la mi-novembre (Charbonneau, Lavoie et Légaré, 1970: 116-117; Olivier, 1975).

3 Robert R. Kuczynski est un démographe britannique qui a notamment publie un ouvrage intitule Birth Registration and Birth Statistics in Canada (1930); c est a cet ouvrage auquel Sabagh fait allusion. 


\section{L'etat de la population et sa structure}

Les auteurs qui font l'objet de cette etude se sont interesses a des degres divers a l'etat et a la structure de la population canadienne. A cet egard, c'est surtout le XVIIe siecle qui a retenu l'attention, a cause de 1 'existence de recensements nominatifs pour les années 1666 , 1667 et 1681 et de l'attrait exercé par les origines de la Nouvelle-France sur les historiens qui les ont précedes. C'est ainsi que, pour connaitre le nombre d'habitants au Canada aux XVIIe et XVIIIe siecles, Langlois a epluché les ouvrages de ses prédecesseurs, ce qui lui permet d'aligner quelques colonnes de chiffres. Reprenant Sulte, l'auteur estime qu'il y avait 675 habitants en 1653 et entre 2000 et 2500 dix ans plus tard. Langlois a ensuite relevé dans Sulte, Salone, Chapais et Renaud le nombre d'individus recenses a certaines dates, de 1666 a 1739. Et comme les donnés de Salone et celles de fienaud ne concordent pas, sauf en 1706, Langlois choisit celles de Renaud parce qu'elles se rapprochent des chiffres de l'accroissement naturel et des statistiques officielles (1935: 73-77). En ce qui concerne la structure de la population selon le sexe, l'age et l'etat matrimonial, l'apport de Langlois est plutat maigre. Il note la supériorité numérique des hommes au XVIIe siecle et demontre par un tableau de chiffres de population (de 1665-1666 [sic] a 1737) la "convergence des sexes", et 1 a tendance a l'équilibre entre les sexes qui s'établit en 1719 (1935: 79-80), ce que confirme du reste un tableau d'Henripin (1954a: 12-21). Langlois ne pousse guere les explications et renvoie plutot son lecteur a "la population de la Nouvelle-France: sexes et etats civils", un appendice qui, selon lui, se passe de commentaires (1935: 244-245).

Henripin traite de la structure de la population dans un chapitre de La Population canadienne au debut du XVIIIe siecle intitule "La conjoncture démographique de 1660 a 1760" (1954a: 12-21). Chez lui, l'etude de cette question est centrée sur les recensements du XVIIe siecle, qu'il commente a l'aide des pyramides des ages qu'il en a tirées. La silhouette de ces pyramides est d'ailleurs tres anormale, surtout en 1666 et 1667 , parce qu'il y a plus d'hommes que de femmes dans la population a ce moment-la et qu'on voit tres bien la superposition des classes d'immigrants par rapport aux Canadiens de naissance, un phénomene dont l'importance tend a se résorber en 1681 . En ce qui concerne la repartition suivant l'etat matrimonial, Henripin note la forte proportion de célibataires masculins agés de 20 ans et plus lqui tient au déséquilibre des sexes), l'existence d'une fraction non négligeable de femmes mariées agées de 16 a 20 ans (fraction qui diminue cependant en 1681 ) ainsi que la proportion minime des veufs et des veuves. L'observation diune série de recensements de 1666 a 1790 lui permet dailleurs de conclure que la proportion normale de célibataires dans cette population est de $60 \%$, contre $40 \%$ de mariés et de veufs (1954a: 17-21). S'estimant satisfait du travail d'Henripin, Biraben $n$ a pas cherché a pousser plus loin l'analyse des structures démographiques; a ce sujet, il ne fait d'ailleurs que signaler quelques traits caractéristiques de la population de la Nouvelle-France et corriger certains chiffres avances par quelques-uns de ses informateurs (1966: 114 et 120$)$. 
En ce qui a trait a la repartition gégraphique de la population, ce qu'on trouve dans Langlois est plutôt décevant: l'auteur ne montre pas comment les hommes ont progressivement occupé le territoire; il s'attache trop a Montréal par rapport au reste de la colonie, il oublie le Richelieu et maltraite la Nouvelle-Beauce, comme nous l'avons déja signalé. Il se contente d'une description sommaire, sans chercher dexplication au mouvement de la population dans lespace (1935:84-86). Henripin, lui, a dressé une carte qui montre 1 étalement de la population sur le territoire, chaque point représentant une paroisse. Cet étalement est dailleurs présenté comme un "éparpillement extravagant" dú au régime seigneurial (1954a: 3,9 et 21). Biraben se trouve en quelque sorte a contrebalancer ces tendances laurentiennes, puisqu'il fait etat du peuplement français dans les Pays-den-haut, aux Illinois et en Acadie (1966: $121-124)$.

\section{Le mouvement de la population}

\section{1) L'immigration et l'émigration}

Grace aux graphiques d'Henripin et de Biraben, on peut suivre dans le temps le mouvement de la population. Sur le mouvement migratoire, Langlois, Henripin et Biraben se sont prononcés. Pour ce qui est du nombre d'immigrants venus au Canada sous le regime français, Langlois, qui emprunte a Renaud son tableau par périodes de vingt ans et par categories sociales, estime qu'il en est venu 10126 (1935: 59-60); Biraben, qui trouve que 1 'on a sous-estimé le nombre d'immigrants entre 1640 et 1679 , avance le chiffre de 11370 (1966: 120). C'est en 1633 que commence vraiment le peuplement du Canada. Precisons que les immigrants sont venus surtout au XVIIe siecle, entre les annés 1654-1659 et 1663-1673 plus spécialement. Au XVIIIe siecle, il vient a peine 50 immigrants par an jusquen 1740 et 180 par an, de 1740 a 1760 , affirme Henripin (1954a: 12). Pour decrire ces gens, celui-ci emprunte une citation a Garneau. Ce sont des aventuriers, ecrit l'historien, des jeunes gens de bonne famille qui revent de faire fortune, des marins las de la vie en mer, des catholiques fuyant le voisinage des huguenots, des soldats licencies ou, enfin, des braconniers et des faux-saulniers (1954a: 13). D où viennent-ils? - Biraben, a partir des fiches de Godbout, a relevé l'origine des filles a marier venues au Canada; il constate que la plupart proviennent de la région de Paris, tres peu du nidi de la France ou de l etranger. Les hommes par contre ont des origines plus variés, comme l'a démontré Ferland (Biraben, 1966: 119; Langlois, 1935: 45-46). En somme, sur cette question, les auteurs ne font pratiquement que répéter ce que les historiens du XIXe siecle avaient écrit.

Au sujet de l'émigration, d'autre part, nous trouvons peu de choses, car ce phénomene a été moins étudié que sa contrepartie. On sait qu'elle a existé, Henripin le note d'ailleurs dans sa these: 
"En fait, on a de bonnes raisons de penser qua XuIIIe siecle, 1 émigration a été au moins aussi forte que l'immigration. De 1700 a 1760,1 'immigration se chiffre 5000 environ. On sait, dautre part, que, de 1720 a 1739,3500 Canadiens ont gagné la Louisiane. Dautres ont dú faire de meme, avant et apres cette période. Il faut encore tenir compte des pertes dues a la course dans les bois. Les coureurs des bois étaient au nombre de deux a quatre mille, vers 1760." (1954a: 75)

Voila certes une catégorie d'individus qui a mauvaise presse. Ainsi, au dire de Langlois, ces insaisissables sont innombrables et constituent un

"élément démographique en quelque sorte négatif puisque les coureurs de bois, célibataires irreductibles pour la plupart, contribuaient a ralentir la natalité en sabstenant du mariage ou en y venant tard, a affaiblir la population en la dispersant et a la diminuer en émigrant." (1935: 81)

Langlois fait aussi état d'immigrants qui rentrent en France de façon definitive, sans pouvoir en évaluer le nombre cependant (1935: 54).

\section{2) La natalite}

La politique de la France ayant été de peupler la colonie sans dépeupler la métropole, le roi misait par conséquent sur l accroissement naturel des Canadiens. A cet egard, "on ne saurait reprocher aux Canadiens dalors de n'avoir pas fait ce qu'ils pouvaient ", comme l'observent Langlois (1935: 192) et Henripin (1968: 2). Sur la natalité au Canada sous le régime français, Langlois est plutôt vague et se borne a quelques idées genérales sur la question; Henripin, plus précis, prend la mesure statistique du phénomene. Langlois a senti qu'il y avait, chez les Canadiens de cette époque, "tendance a la natalitémaxima", ce que viennent illustrer de nombreux témoignages de contemporains. A le lire, on a l'impression que la colonie etait remplie de familles nombreuses (1935: 103-114). Malheureusement, l auteur ne nous fait pas prendre conscience des variations annuelles ou saisonnieres du nombre de naissances: il faut se reporter aux statistiques de l'etat civil pour cela ou encore a la these d'Henripin.

Celui-ci a suivi dans le temps le mouvement des naissances. Il a constaté a l'occasion quelques brisures dans la pente ascendante de la natalite, pendant les guerres et les épidénies en particulier (1954a: 16-17); il a calculé des taux de natalité par décennie qu'il commente prudement: selon lui, aprés 1710 , ces taux ne sont pas gonflés par une structure de population anormale (il y avait au Canada, avant cette date, surreprésentation masculine et peu de vieillards); "il semble bien assuré que cette population jouissait d'une natalité exceptionnelle" ajoute-t-il. A l'aide de Tanguay, Henripin a examiné le mouvement saisonnier des naissances; les premieres naissances dépendent le plus souvent de la date du mariage: pour les suivantes, en décalant de neuf mois la date de naissance, Henripin a pu oresser la courbe du mouvement saisonnier des conceptions (1954a: 122), qu'il donne comme exemple de 
soumission au rythme de la nature. Il a egalement remarqué une hausse croissante du taux de naissances illegitimes entre 1700 et 1760 . Il note enfin que ce sont des familles de huit, cinq et six enfants que l on trouvait le plus souvent. La taille des familles etait en effet limitée, malgré une forte fecondite, par l'age relativement avancé de la femme lors de son mariage ainsi que par le déces assez fréquent de l un des époux avant que la femme natteigne cinquante ans (1954a: 51).

3) La nuptialite

En consultant les mémes auteurs sur la question de la nuptialité, nous obtenons deux images tres différentes de ce phenomene. Langlois, qui s'est ici surtout inspiré de Tanguay, en fait un récit plutót anecdotique où les cas particuliers risquent de passer pour la regle genérale. L auteur se contente de dire que pour qu'il y ait accroissement rapide de la population de la Nouvelle-France, il fallait que les mariages fussent hatifs, nombreux et féconds. Il rapporte par ailleurs que les femmes etaient tres recherchés: les filles a peine puberes et les veuves trouvaient facilement parti (ce qui a été dementi par Henripin). Langlois parle aussi des annulations de mariage, des mariages a la gaumine et des mariages franco-indiens (1935: 87-102), questions qui ont soulevé l'intéret des curieux a la fin du XIXe sieclé.

Henripin, pour sa part, mesure le phénomene et tente quelques explications sur les pointes et les creux quil releve sur la courbe de nuptialite (1954a: 15-16). Les taux de la période 1700-1730 comportent peut-etre une marge derreur qui serait due a notre méconnaissance des mouvements migratoires de 1 époque, avoue-t-il. Le mouvement saisonnier des mariages correspond, selon lui, au rythme de la vie rurale - $54 \%$ des mariages ont lieu en novembre, février et janvier. Sur les caracteristiques des conjoints, Henripin detruit certains mythes, notamment celui du mariage precoce des filles. L'auteur a dressé un tableau de l'age moyen au mariage, selon les categories d'unions (1954a: 96). Four l'age modal, Henripin constate que les hommes se marient le plus souvent a vingt-six ans et les femmes a vingt ans. Quant au veuvage, il dure en moyenne deux ans pour les hommes et trois ans pour les femmes. Les veuves n'etaient donc pas aussi recherchés au XVIIIe siecle qu'au XVIIe. Les résultats demontrent, en outre, que les hommes - celibataires et veufs epousent moins souvent une veuve que les femmes un veuf. Henripin est porté a croire quil y avait moins de veuves que de veufs au Canada entre 1700 et 1730 , a cause du nombre de meres qui mouraient en couche (1954a: 95). L'auteur conclut que "la population canadienne avait au XVIIIe siecle un attrait prononce pour la vie conjugale"(1954a: 102).

- Lexpression "mariages a la gaumine" désigne certains mariages qui ne se faisaient pas dans les formes prescrites par leglise. Tout a fait exceptionnels, ces cas ont été dénonces par les autorités ecclesiastiques. 


\section{4) La fécondité}

Sabagh et Henripin sont les deux seuls cependant a avoir etudiéla fécondité de la population canadienne sous le régime français. Sabagh est arrive a démontrer, en dépit de l'imperfection de ses sources, que la forte fécondité des Canadiennes au XVIle siecle n'est pas un mythe, car la grande majorité des femmes alors en age de procreer etaient mariees (1942: 689). Henripin, qui s'est penché au début de sa carriere sur les trois premieres décennies du XVIIIe siécle et qui a, par la suite, continuéd'étudier le phénomene de la fécondité, écrit que les couples canadiens du XVIIIe siecle jouissaient de l'une des plus fortes fécondités qui aient été mesurées jusqu'a ce jour, mais que celle-ci n'est peut-etre pas aussi exceptionnelle qu'on a pu le penser (1968: 11). Henripin a constate que la fécondité féminine ne baisse que tres lentement entre vingt et quarante ans et qu'au-dela de cet age, la chute s'accélère. Les ménages canadiens auraient ainsi eu en moyenne 13,27 enfants, si la femme s'était mariée a quinze ans et était demeurée dans cet etat jusqu'a cinquante ans. Or, en réalité, les femmes se mariaient dans la vingtaine et risquaient fort de mourir ou de devenir veuves avant l'age de cinquante ans. C'est ce qui explique que les couples étudies par Henripin avaient en moyenne une descendance finale de 5,65 enfants. L'intervalle entre les naissances était de 23,3 mois en moyenne; entre le mariage et la premiere naissance, il etait cependant de 16,8 mois. On aurait donc tort de croire qu'il s'ajoutait un enfant tous les ans dans les familles de nos ancetres (1954a: 61).

\section{5) La mortalité}

L'un des aspects les moins etudiés de l'évolution de la population canadienne est certainement le phénomene de la mortalité. Cela tient au sous-enregistrement des déces, qui fait que le déces des individus est le renseignement qui manque le plus souvent dans Tanguay: c'est pourquoi 1'échantillon d'Henripin ne comporte que les deux cinquiemes des déces des parents et les deux tiers de ceux des enfants (1954a: 63). Henripin ne s'est donc pas risque dans une étude de la mortalité par age, estimant les données probablement faussés. Il s'est ainsi limité a une étude de la mortalité infantile. L'auteur s'est rendu compte par un jeu mathématique que les enfants morts apres un an ont échappé aux investigations de Tanguay dans une proportion beaucoup plus forte que les enfants morts avant un an. Il estime que 246 enfants sur 1000 sont morts au cours de leur premiere annee d'age, ce qui vient démentir les assertions de Langlois sur la faiblesse de la mortalite infantile (Henripin, 1954a: 106; Langlois, 1935: 123).

Dans l'ensemble, on s'est borné des constatations trés générales sur la mortalite. Henripin a relevé des pointes de mortalité. Elles sont dues aux guerres ou aux epidémies: petite vérole, c'est-a-dire variole, appelé aussi grande picotte; maladie des navires, c'est-a-dire typhus, appelé aussi fievre pourprée. Toutefois, on est assez mal renseigné sur les causes de mortalité en temps ordinaire puisque ce sont surtout des déces de nature accidentelle qui 
sont consignés dans les registres: victimes des Iroquois, d'accidents de travail ou de noyades. C'est d'ailleurs sous toutes réserves et sans commentaires que Langlois cite Tanguay a ce propos (1935: 126).

\section{La representation graphique des phenomenes de population}

Les cartes et graphiques sont des images qui decrivent la réalité et nous la font saisir d'une façon globale, ce qui des lors facilite la compréhension. Depuis l'epoque de Langlois, il y a certainement eu evolution de l'opinion sur l'utilisation des ces moyens. Dans l'Histoire de la population canadienne-francaise, il n'y a ni cartes, ni graphiques et les tableaux de chiffres ont ete rejetés en appendice. Dans La Population canadienne au debut du XVIIIe siecle, il y a vingt graphiques en appendice portant sur l'ensemble des phenomenes etudiés; la moitie d'entre eux concernent cependant la fécondite. On ne trouve toutefois qu une seule carte dans cet ouvrage (1954a: 22 ) et l'auteur aurait dú expliquer par une légende que chaque point représente une paroisse ayant fourni des actes a l'etude. Dans son artirle dans Population et dans sa "Breve histoire demographique..." Henripin a inclus un certain nombre de graphiques (1954b: $67,70,72,77$ et $79 ; 1968$ : $4,6,9$ et 12 ). Quant a l'article de Biraben, il ne contient pas de carte sur la population canadienne sous le régime français, mais seulement pour la période postérieure. Il donne cependant un graphique de l'évolution comparée des populations d'Amérique du Nord (1966: 115).

\section{Les idees et les politiques demographiques}

Avec son "essai d'analyse démographique", Langlois est le seul parmi les auteurs étudies a s'etre intéresse aux idées et aux politiques démographiques du régime français (1935: 185-240). L'intitule de la deuxieme partie de l'Histoire de la population canadienne-française démontre cependant que le vocabulaire de la démographie n'était pas encore fixédans les annés trente: on ne parle aujourd'hui d'analyse démographique que pour l'étude des phénomenes de population eux-memes et non pour celle des idees. Langlois a certainement subi ici l'influence de son maître Gonnard qui avait lui-meme écrit sur les idées et les politiques démographiques. Malgré son originalité, l'étude de Langlois est cependant un peu faible parce qu'elle s'appuie sur des bases limitees. On en sort avec une image aujourd'hui vieillie de la grandeur de la France au XVIIe siecle et une vision tres noire de la politique coloniale britannique. Un article recent de Charbonneau et Landry (1979) vient heureusement de refaire le point sur cette question.

\section{CONCLUSION}

Au terme de ce travail, certains traits se dégagent de l'ensemble des textes que nous avons examines, tous publies entre 1934 et 1966 . Notre analyse par themes a démontré que divers aspects de la vie de la population canadienne ont été abordés et que quelques-uns ont été mieux traités que d'autres, soit en quantite, soit en qualité. Les questions les plus négligés paraissent etre les migrations et la mortalité. Bien qu'ils aient fait couler beaucoup 
dencre, les immigrants étaient encore mal connus en 1966: leur nombre, par exemple, faisait et fait toujours l'objet de discussions. L'émigration a éveille encore moins de curiosité: le phénomene n'a guere été mesuré; on se contente tout juste de dénoncer les départs temporaires ou definitifs des coureurs de bois. Quant aux migrations interieures, il n'en a pas eté question. Sur la mortalité, on a peu écrit; ici les sources sont defectueuses et leur critique systématique s'impose. La mortalité génerale n'a pas été mesurée avec précision sinon par des taux bruts décennaux. Henripin a donc été prudent en ne traitant que de la mortalité infantile. Par contre, d'autres sujets ont fait l'objet de recherches plus elaborés: la population aux recensements du xVIIe siecle, la natalité, la nuptialité et la fécondité. Henripin a fait une étude remarquable de ces trois phenomenes et son étude demeure utile, meme apres la publication de Vie et ort de nos ancetres par Charbonneau en 1975. I1 a notanment introduit la mesure là où Langlois n'avait recueilli que des impressions generales. Sur la structure de la population - aux recensements de 1666, 1667 et 1681 , en particulier - les idées analysées ici sont maintenant depassees. Plusieurs articles du programme de recherhe en démographie historique parus apres 1966 ont fait progresser les connaissances sur ce sujets"

Dans toute cette production, il faut donner a chacun selon son mérite. André Vachon (1962) a dit de l'etude de Langlois qu'elle est assez faible parce qu'elle ne repose pas sur une etude critique des chiffres fournis par les recensements ou les documents officiels. Le jugement est juste mais un peu rapide. En fait, il ne souligne pas l'originalité de l'oeuvre de Langlois. Cette premiere synthese d'histoire de la population constitue pourtant, rappelons-le, "l'acte de naissance de la recherche démographique sur le Canada franças" et, au dire d'Henripin, son contenu fait passer la demographie des Canadiens français de la mythologie a la connaissance empirique (1962: 133 ). Lorsqu'il se penche sur le régime français, Langlois n'est peut-être pas tres créateur. Mais sa contribution n'est pas inutile: en organisant en un tout coherent un savoir jusque-la dispersé, Langlois donne a la recherche la premiere synthese dont elle a besoin pour aller plus loin dans le domaine de la démographie du passé. Viennent ensuite les analyses: coups de sonde limites dans le temps, les travaux de sabagh et d'Henripin précisent les contours definis par Langlois. Par sa methode, la these d'Henripin - qui est l'un des classiques de la demographie - marque un net progres. Enfin, dans une synthese plus rapide que celle de Langlois, Biraben, meme s'il nous laisse insatisfait sur certains points, rajeunit la presentation du "peuplement du Canada franças". Depuis 1966, cependant, les progrès de la recherche sur la population canadienne sous le régime français ont eté considerables. C'est un autre long rapport qu'il faudrait pour en rendre compte.

- Voir notamment: Charbonneau et Legaré, 1967; Charbonneau, Lavoie et Legaré, 1970 et 1971; Charbonneau et Lavoie, 1971; 0livier-Lacamp et Légaré, 1979. 


\section{RÉFÉRENCES BIBLIOGRAPHIQUES}

BIRABEN, Jean-Noël, 1966. "Le peuplement du Canada français". Annales de dérographie historique, $105-138$.

CANADA, ministere de l'Agriculture, 1876. Recensenent du Canada, 1871, vol. IV, statistiques du Canada. Ottawa, I.B.Taylor, 422 p.

CANADA, ministere de l'Agriculture, 1878. Recensement du Canada, 1871, vol.v, Statistiques du Canada. Ottawa, Maclean, Roger \& Co., $479 \mathrm{p}$.

CHARBONNEAU, Hubert, 1966. "Tricentenaire du premier recensement canadien". Population, 21, 6, 1211-1215.

CHARBONNEAU, Hubert, 1975. Vie et ort de nos ancetres. Etude démographique. Montréal, Les Presses de l'Université de Montreal, 268 p. (Coll. "Démographie canadienne", no 3 ).

CHARBONNEAU, Hubert et a1., 1967. "La démographie historique au Canada: un projet de recherche". Recherches sociographiques, VIII, 2, 214-217.

CHARBONNEAU, Hubert et LANDRY, Yves, 1979. "La politique démographique en Nouvelle-France". Annales de déographie historique, 29-57.

CHARBONNEAU, Hubert et LAVOIE, Yolande, 1971. "Introduction a 1 a reconstitution de la population du Canada au XVIIe siecle. Etude critique des sources de la période 1665-1668". Revue d'histoire de I'A érique française, 24, $4,485-511$.

CHARBONNEAU, Hubert, LAVOIE, Yolande et LEGARE, Jacques, 1970. "Recensements et registres paroissiaux du Canada durant la période 1665-1668. Etude critique". Population, 25, 1, 97-124.

CHARBONNEAU, Hubert, LAVOIE, Yolande et LEGARE, Jacques, 1971. "Le recensement nominatif du Canada en 1681". Histoire sociale/Social History, 7, 77-98.

CHARBONNEAU, Hubert et LEGARE, Jacques, 1966. "Les sources démographiques de la Nouvelle-France au XVIIe siecle. Présentation d'un projet de recherche". Communication présentée au 4 e congrés de l'ACFAS, novembre 1966.

CHARBONNEAU, Hubert et LEGARE, Jacques, 1967. "La population du Canada aux recensements de 1666 et 1667". Population, 22, 6, 1031-1054.

HENRIPIN, Jacques, 1954a. La Population canadienne au debut du XVIIIe siecle. Nuptialité - fecondité - ortalité infantile. Paris, Presses universitaires de France, 129 p. (I.N.E.D., coll. "Travaux et documents", cahier no 22 ).

HENRIPIN, Jacques, 1954b. "La fécondité des ménages canadiens au début du XVIIIe siecle". Population, 9, 1, 61-82. 
HENRIPIN, Jacques, 1962. "Les etudes demographiques" in F. Dumont et Y. Martin, situation de la recherche sur le Canada francais. Quebec, Presses de l'Université Laval, 133-144.

HENRIPIN, Jacques, 1968. Tendances et facteurs de la fecondite au Canada. ottawa, Bureau féderal de la statistique, $425 \mathrm{p}$.

HENRY, Louis, 1956. Anciennes fanilles genevoises, etude denographique: seizilee-vingtieae silcles. Paris, Presses universitaires de France, $235 \mathrm{p}$. (I.N.E.D., coll. "Travaux et documents", cahier no 26).

HENRY, Louis, 1973. "La demographie au service de l'histoire", in Honage a Harcel Reinhard. Sur la population francaise au XVIIe et au XVIlle siecles. Paris, Sociéte de démographie historique, 341-342.

KUCZYNSKI, Robert R., 1930. Birth Registration and Birth Statistics in Canada. Washington, D.C., Brookings Institution, $226 \mathrm{p}$.

LANGLDIS, Georges, 1935. Histoire de la population canadienne-francaise. 2e éd. Montréal, Albert Lévesque, $309 \mathrm{p}$.

LANGLOIS, Georges, 1938. "Essai sur le peuplement de l'Anerique: introduction a une étude du peuplement du Canada". L'Actualite Conosique, XIV, t.2, 1, 1-16.

LANGLOIS, Georges, 1939. "Essai sur le peuplement de l'Amerique: introduction a une etude du peuplement du Canada". L'Actualite econoaique, XIV, t.2, 5 , 440-453.

OLIVIER, Gaël, 1975. "Determination du moment du recensement nominatif de 1681", in Programme de recherche en demographie historique, Rapport annuel 1974-1975. Département de démographie, Université de Montréal, 83-89.

OLIVIER-LACAMP, Gaël et LEGARE, Jacques, 1979. "Quelques caractéristiques des ménages de la ville de Quebec entre 1666 et 1716". Histoire sociale/Social History, XII, 23, 66-78.

SABAGH, Georges, 1942. "The Fertility of the French Canadian Women During the Seventeenth Century". Aaerican Journal of Sociology, XLVII, 5, 680-689.

SALONE, Enile, 1905 et 1970. La colonisation de la Nouvelle-france. Paris, Guilmoto; réimpr.: Trois-Rivières, Boréal Express, $505 \mathrm{p}$.

SAUVY, Alfred, 1954. "Présentation", in J. Henripin, La Population canadienne au debut du Xville siecle. Nuptialite - fecondite - cortalite infantile, Paris, Presses universitaires de France, xi-xiii. (I.N.E.D., coll. "Travaux et documents", cahier no 22).

VACHON, André, 1962. "Etat des recherches sur le régime français (1632-1760)", in F.Dumont et Y. Martin, Situation de la recherche sur le Canada francais, Québec, Presses de l'Université Laval, 11-24. 


\section{REBUHE - BUMMARY - REBUNEN}

\section{LaROSE André - Les denographes ot la population du Canade sous le ropien francals (1934-1966)}

Avant que les dénographes Hubert Charbonneau et Jacques Légaré ne lancent leur vaste enquete sur 1 a population de la Nouvelle-france au nilieu des annees soixante, d'autres denographes s'étaient penches sur l'histoire de cette atee population: Georges Langlois en 1934, Georges Sabagh en 1942, Jacques Henripin en 1954 et Jean-Noël Biraben en 1966. On trouvera ici un coupte rendu de leurs travaux. Apress avoir présenté l'une après l'autre leurs publications respectives, l'auteur évalue leur contribution et net en relief les forces et faiblesses de chacun, en abordant tour a tour les sources et la critique des sources, l'état de la population et sa structure, le nouvenent de la population et ses conposantes et enfin, les idées et les politiques dénographiques.

\section{LaROSE Andre - Denographers and the Population of Canade undar the Froneh Region (1934-1966)}

Prior to Hubert Charbonneau's and Jacques Légare's extensive survey on the population of Nen France, initiated in the nid-sixties, other denographers had looked into the history of this population: Georges Langois (1934), Georges Sabagh (1942), Jacques Henripin (1954) and Jean-Noël Biraben (1966). This article presents a review of their works. After presenting their respective publications, the author evaluates their contribution, and highlights the strengths and meaknesses of each, exanining successively the sources and the assessment of the sources, the conposition and structure of the population, the population changes and their components, and finally, the population policies and ideas.

\section{LaROSE André - Los denógrafos y la población de Canadi durante el régian franchs (1934-1966)}

Antes de que los denógrafos Hubert Charbonneau y Jacques Légaré desplegaran su anplia investigación sobre la población de la Nueva Francia a mediados de los sesentas, otros denógrafos se habían ya interesado en la historia de esta nisaa población: Georges Langlois en 1934, Georges Sabagh en 1942, Jacques Henripin en 1954 y Jean-Noël Biraben en 1966. El lector encontrará aqui una reseña de sus trabajos. Después de presentar cada una de sus publicaciones, el autor valoriza su contribución y pone de anifiesto la robustez y las flaquezas de cada uno, abordando, a su vez, las fuentes y su crítica, el estado de la población y su estructura, el moviaiento de la población y sus conponentes $y$, por últino, las ideas y las políticas denográficas. 